\title{
Lack of Mutations in POT1 Gene in Selected Families with Familial Non-Medullary Thyroid Cancer
}

\author{
Aida Orois ${ }^{1,2}$ (1) Celia Badenas $3,4,5$. Jordi L. Reverter ${ }^{6}$. Verónica López ${ }^{3,4,5} \cdot$ Miriam Potrony ${ }^{3,4,5} \cdot$ Mireia Mora ${ }^{1,4,7,8}$. \\ Irene Halperin ${ }^{1} \cdot$ Josep Oriola ${ }^{3,4,7}$
}

Received: 24 December 2019 / Accepted: 4 March 2020 / Published online: 14 March 2020

(C) Springer Science+Business Media, LLC, part of Springer Nature 2020

\begin{abstract}
To date, the genes involved in familial non-medullary thyroid cancer (FNMTC) remain poorly understood, with the exception of syndromic cases of FNMTC. It has been proposed that germline mutations in telomere-related genes, such as POT1, described in familial melanoma might also predispose individuals to thyroid cancer, requiring further research. We aimed to identify germline mutations in POT1 in selected FNMTC families (with at least three affected members) without a history of other cancers or other features, and to describe the clinical characteristics of these families. Sequencing of the 5'UTR and coding regions of POT1 was performed in seven affected people (index cases) from seven families with FNMTC. In addition, we performed whole-exome sequencing (WES) of DNA from 10 affected individuals belonging to four of these families. We did not find germline variants of interest in POT1 by Sanger sequencing or WES. We neither found putative causative mutations in genes previously described as candidate genes for FNMTC in the 4 families studied by WES. In our study, no germline potentially pathogenic mutations were detected in POT1, minimizing the possibilities that this gene could be substantially involved in non-syndromic FNMTC.
\end{abstract}

Keywords POT1 $\cdot$ Germline $\cdot$ Telomere $\cdot$ Familial non-medullary thyroid cancer

\section{Introduction}

Thyroid cancer (TC) is one of the most frequent cancers, and its prevalence is increasing in the last years [1]. TC may originate from follicular or parafollicular cells, referred as nonmedullary thyroid carcinoma (NMTC) or medullary thyroid carcinoma (MTC), respectively. A majority of thyroid cancer cases $(80-90 \%)$ originate from follicular cells, and $3-9 \%$ of them present first-degree relatives with NMTC $[2,3]$.

Aida Orois

aorois@clinic.cat

1 Department of Endocrinology and Nutrition, ICMDM, Hospital Clinic de Barcelona, C/Villarroel 170, 08036 Barcelona, Spain

2 Department of Endocrinology and Nutrition, Hospital Universitari Mútua de Terrassa, 08221 Terrassa, Spain

3 Department of Biochemistry and Molecular Genetics, CDB, Hospital Clínic de Barcelona, 08036 Barcelona, Spain

4 Institut d'Investigacions Biomèdiques August Pi i Sunyer (IDIBAPS), 08036 Barcelona, Spain
Familial non-medullary thyroid cancer (FNMTC) is defined by the presence of thyroid cancer originating from follicular cells in two or more first-degree relatives, in the absence of predisposing environmental factors. Five percent of all FNMTC cases are syndromic and the susceptibility genes involved in syndromic FNMTC are known: $A P C$ in familial adenomatous polyposis (MIM: 175100), PTEN in Cowden's disease (MIM: 158350), PRKARIA in Carney complex type 1 (MIM: 160980), WRN in Werner's syndrome (MIM: 277700),

5 Centro de Investigación Biomédica en Red de Enfermedades Raras (CIBERER), Instituto de Salud Carlos III (ISCIII), 28029 Madrid, Spain

6 Department of Endocrinology and Nutrition, Germans Trias i Pujol Health Science Research Institute and Hospital, Universitat Autònoma de Barcelona, 08196 Badalona, Spain

7 Faculty of Medicine, University of Barcelona, 08007 Barcelona, Spain

8 Centro de Investigación Biomédica en Diabetes y Enfermedades Metabólicas Asociadas (CIBERDEM), Instituto de Salud Carlos III (ISCIII), 28029 Madrid, Spain 
and DICER1 in the DICER1 syndrome (MIM: 606241) [4]. However, most cases of FNMTC (95\%) are non-syndromic and the genetic causes are still unknown [5]. Thus far, different strategies have been carried out in order to find out the genes involved in the development of FNMTC: linkage [6, 7], GWAS [8], and next-generation sequencing studies $[9,10]$. Several candidate regions such as PRN (1q21), NMTC1 (2q21), FTEN (8p23), MNG1 (14q32), and TCO (19p13.2), as well as candidate genes like SRGAP1, NKX2, FOXE1, $H A B P 2$, or $M A P 2 K 5$, have been suggested, but none clearly validated as causative of FNMTC [11-13].

It has long been known that somatic mutations in the TERT promotor gene are involved in the development of TC [14], suggesting that genes associated with, or taking part of the telomerase complex, could be involved in the development of non-syndromic FNMTC. Some studies have already been carried out in TC, e.g., TERC gene [15], showing no germline mutations in this gene.

POT1 (protection of telomeres 1) gene is located in chromosome 7 and it is a member of the shelterin complex, encoding a nuclear protein involved in telomere maintenance. Germline variants in POT1 have been described mainly not only in families with melanoma [16], but also in familial gliomas and Li-Fraumeni-like familial cancer syndromes [17, 18]. Recently, it has been suggested that germline mutations in POT1 gene could be implicated in TC, specifically the new variant p.(Lys90Glu) of POT1 identified by whole-exome sequencing (WES) in a family with multiple family members affected with melanoma as well as thyroid, kidney, and breast cancers [19]. In another family with melanoma, a case of TC has been recently reported in an individual with the p.(Ile78Thr) mutation [20]. On the other hand, it has been described that the spectrum of cancers caused by mutations in the POT1 gene is more diverse than it seemed at first [21]. Therefore, with the purpose of reinforcing or not if TC is part of the mutational POT1 phenotype spectrum, it would be interesting to know if mutations in these gene could lead to nonsyndromic FNMTC. In order to elucidate this possibility, we analyzed the POT1 gene in seven selected families, each of them with three or more affected cases to minimize the possibility that thyroid cancer could have been due to chance in these families.

\section{Methods}

\section{Patients}

We designed a multicentric study in Spain to collect blood specimens ( $15 \mathrm{ml}$ of whole blood in potassium EDTA tubes), and clinical data from families with at least three first-degree relatives affected with NMTC, confirmed by histology, without history of other malignancies, and without clinical characteristics suggestive of syndromic FNMTC. We obtained blood samples and clinical data from the affected individuals belonging to seven families with non-syndromic FNMTC, from seven hospitals in Spain (Fig. 1). Patients gave written informed consent before undergoing evaluation and testing.

\section{DNA Extraction and Gene Sequencing}

Genomic DNA was extracted from peripheral blood samples using conventional salt-precipitation protocol. The whole coding region and 5'UTR regions of POT1 gene (NM_015450.2), as well as intron-exon boundaries, were studied.

PCR followed by Sanger sequencing was performed. PCR conditions were as follows: denaturation at $95{ }^{\circ} \mathrm{C}$ for $5 \mathrm{~min}$, 10 cycles $\left(95^{\circ} \mathrm{C}\right.$ for $1 \mathrm{~min}, 65-60{ }^{\circ} \mathrm{C}$ for $1 \mathrm{~min}, 72^{\circ} \mathrm{C}$ for $1 \mathrm{~min}$ ), followed by 25 cycles $\left(95^{\circ} \mathrm{C}\right.$ for $1 \mathrm{~min}, 55^{\circ} \mathrm{C}$ for $1 \mathrm{~min}, 72{ }^{\circ} \mathrm{C}$ for $\left.1 \mathrm{~min}\right)$, and extension at $72{ }^{\circ} \mathrm{C}(10 \mathrm{~min})$. Sanger sequencing was performed using universal M13 primers by GENEWIZ (Takeley, UK). Sequences were analyzed using the SeqPilot 4.0.1 software (JSI Medical Systems, Ettenheim, Germany). POT1 sequencing was performed in seven affected individuals (the index cases) from seven different pedigrees with FNMTC.

\section{Exome Capture and Next-Generation Sequencing}

Whole-exome sequencing (WES) was performed in 10 affected individuals (marked with an asterisk in Fig. 1) from the first four families we recruited (kindreds 1,2,3, and 4).

The DNA library was prepared using the SureSelect exon v5-post kit (Agilent Technologies, Santa Clara, CA, USA) that enables the capture of target sequence of exonic regions in the human genome. The libraries were sequenced using the Illumina HiSeq 2000 sequencer (Macrogene, Seoul, South Korea) with 101-base pair (bp) average read length.

\section{Results}

\section{Families}

Regarding the whole data (Table 1) for the 7 pedigrees (in total, 24 FNMTC-affected members), their median age at diagnosis was 41 years (range 23-61 years), 54\% female. Twenty out of $24(83 \%)$ patients presented classic papillary thyroid cancer (PTC), and 17\% follicular variant PTC. Tumor multifocality was present in $46 \%$ of cases and not present in $37 \%$ (in the remaining $17 \%$, we did not have this data). In total, $29 \%$ of tumors were bilateral (46\% unilateral) and $37 \%$ of tumors presented gross extrathyroidal extension and local invasion. Lymph node metastasis was found in 10 cases $(41.6 \%)$, and distant metastasis was present in 2 cases $(8.3 \%)$. One of the patients with distant metastasis died of 
Fig. 1 Families with nonsyndromic FNMTC recruited. Index cases are indicated with black arrows. Asterisk: Affected individuals where the WES was performed. Gray circles: Patients affected with thyroid cancer
Family 1

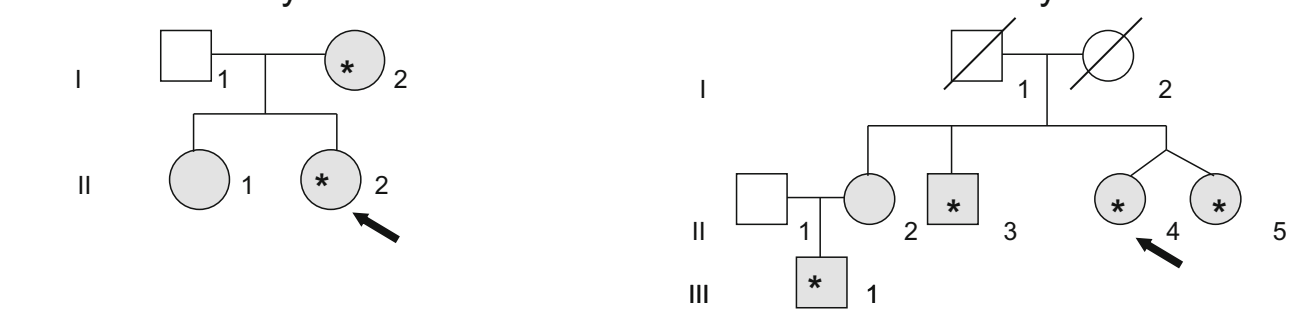

Family 2

Family 3
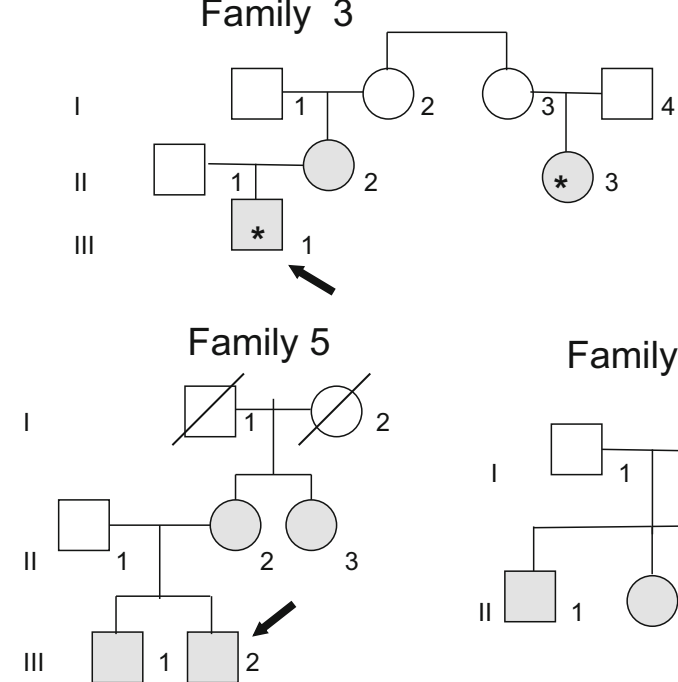

Family 6
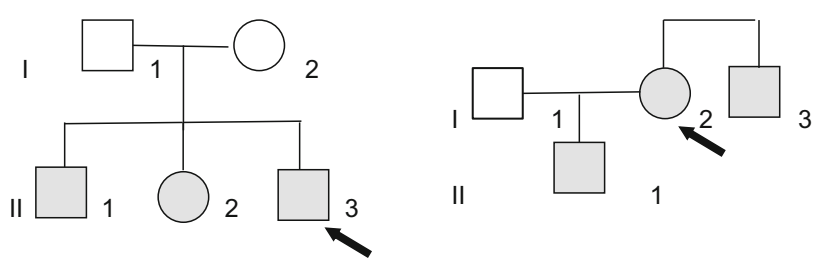

thyroid cancer. All patients received radioactive iodine therapy (range $30-450 \mathrm{mCi}$ ), and to date $29 \%$ presented recurrences and persistence of the disease.

\section{POT1 Analysis}

None of the seven index members from FNMTC was found to be a carrier of POT1 mutations that could be interpreted as causative of TC or other neoplasia. During the analysis, only the following well-known benign changes were found: rs6959712, rs6977407, rs 7794637, rs3815221, and rs142780416. All of them are located in intronic regions.

\section{Whole-Exome Sequencing}

Confirming the previously described sequencing results, none of the 10 subjects studied by WES was found to be a carrier of germline POT1 mutations. In addition, we looked for mutations in other genes related to FNMTC. According to the literature, we examined genes responsible for syndromic FNMTC (APC, PTEN, WRN, DICER1, and PRKAR1A), rearranged genes (NTRK, PPARG), genes involved in DNA repair (XRCC1, XRCC3,ATM), or in the development of the thyroid gland (PAX8,JAG1, CDC42, GSTM1, GSTT1, SRGAP1, TERT, THRB, AKT1, SEC23B, ESR2, NKX2, $T B L 1 X)$, genes previously described that may predispose to non-syndromic FNMTC (HABP2, TTF1, THADA, SEC23B, FOXE1, KLLN, MAP2K5, CHEK2, MYH9, SRRM2), protooncogenes (RET, MET, KIT, MERTK), and genes with somatic mutations in TC (BRAF, NRAS, TP53, CDKN2A, ALK, $A T F 4$ ). We did not find any genetic variant that could be considered causative of thyroid cancer.

\section{Discussion}

Regarding the rising incidence of TC in general, and familial TC in particular, there is an unmet need to identify the genetic risk factors associated with this disease. Many studies have been carried out in order to identify susceptibility genes involved in non-syndromic FNMTC. Numerous reports have found private mutations in specific genes in isolated families, suggesting them to be causative of FNMTC [22], but none of them has been validated across different research articles [23].

$\mathrm{TC}$ and melanoma are in a way genetically connected cancers since they share some features: both can be triggered by environmental factors such as radiation or ultraviolet exposure, suggesting that DNA repair genes may be involved in their pathophysiology [24]. Furthermore, the elevation of TC risk in patients with melanoma has been described [25], and both are observed in a syndrome such as PTEN hamartomatous tumor syndrome (Cowden disease). Interestingly, like melanoma, TC often 
Table 1 Clinical characteristics, pathological findings, and outcome of 7 families with FNMTC (members according to Fig. 1)

\begin{tabular}{|c|c|c|c|c|c|c|c|c|c|c|}
\hline Family & Member & Age (years)/sex & Disease presentation & Type & Tumor multifocality & Bilaterality & Gross ETE & $\mathrm{LN}$ & Distant M1 & Outcome \\
\hline \multirow[t]{3}{*}{1} & I.2. & $26 / \mathrm{F}$ & Unknown & CPTC & No & No & Yes & No & No & Unknown \\
\hline & II.1. & $29 / \mathrm{F}$ & Nodule & CPTC & UK & UK & UK & No & No & Remission \\
\hline & II. 2 . & $31 / \mathrm{F}$ & Nodule & CPTC & Yes & Yes & No & No & No & Persistence \\
\hline \multirow[t]{5}{*}{2} & II. 2 . & $53 / \mathrm{F}$ & Multinodular goiter & CPTC & No & No & No & No & No & Remission \\
\hline & II.3. & $57 / \mathrm{M}$ & Multinodular goiter & CPTC & No & No & Yes & No & No & Remission \\
\hline & II. 4 . & $51 / \mathrm{F}$ & Multinodular goiter & CPTC & Yes & Yes & Yes & No & No & Remission \\
\hline & II.5. & $50 / \mathrm{F}$ & Multinodular goiter & CPTC & No & No & No & No & No & Remission \\
\hline & III.1. & $36 / \mathrm{M}$ & screening & CPTC & Yes & Yes & Yes & Yes & No & Remission \\
\hline \multirow[t]{3}{*}{3} & II.2. & $48 / \mathrm{M}$ & Nodule & СРTC & Yes & No & No & UK & No & Remission \\
\hline & II.3. & $41 / F$ & Nodule & CPTC & No & No & Yes & Yes & No & Persistence \\
\hline & III.1. & $23 / \mathrm{M}$ & Nodule & FPTC & UK & UK & UK & UK & No & Remission \\
\hline \multirow[t]{3}{*}{4} & II.1. & $55 / \mathrm{M}$ & Adenopathy & CPTC & UK & UK & UK & Yes & No & Remission \\
\hline & II. 2. & $61 / \mathrm{H}$ & Adenopathy & CPTC & UK & UK & UK & Yes & Yes & Exitus \\
\hline & III.1. & $37 / F$ & Nodule & CPTC & Yes & No & UK & Yes & No & Persistence \\
\hline \multirow[t]{4}{*}{5} & II. 2 . & $37 / F$ & Nodule & CPTC & No & No & Yes & No & No & Remission \\
\hline & II. 3. & $41 / \mathrm{F}$ & Nodule & FPTC & No & No & No & No & No & Remission \\
\hline & III.1. & $24 / \mathrm{M}$ & Screening & CPTC & Yes & No & No & No & No & Remission \\
\hline & III. 2. & $30 / \mathrm{M}$ & Nodule & FPTC & No & No & No & No & No & Remission \\
\hline \multirow[t]{3}{*}{6} & II.1. & $29 / \mathrm{M}$ & Nodule & CPTC & Yes & UK & Yes & Yes & No & Persistence \\
\hline & II. 2 . & $45 / \mathrm{F}$ & Nodule & CPTC & Yes & Yes & Yes & Yes & No & Remission \\
\hline & II.3. & $43 / \mathrm{M}$ & Nodule & FPTC & No & No & Yes & No & No & Remission \\
\hline \multirow[t]{3}{*}{7} & I. 2 . & $46 / \mathrm{F}$ & Multinodular goiter & CPTC & Yes & Yes & No & Yes & No & Persistence \\
\hline & I.3. & $45 / \mathrm{M}$ & Adenopathy & CPTC & Yes & Yes & No & Yes & Yes & Persistence \\
\hline & II.1. & $36 / \mathrm{M}$ & Nodule & CPTC & Yes & Yes & No & Yes & No & Remission \\
\hline
\end{tabular}

$F$, female; $M$, male; $C P T C$, classic papillary thyroid cancer; $F P T C$, follicular variant papillary thyroid cancer; $E T E$; extrathyroidal extension; $L N$, lymph node metastasis; $M 1$, metastasis; $U K$, unknown

harbors somatic promotor mutations in the TERT gene [26] or the p.Val600Glu mutation in the $B R A F$ gene, both being important events in TC progression [27]. Moreover, it has been suggested that genes involved in telomere maintenance that predispose to develop melanomas may also be implicated in TC, as it has been described that patients with FNMTC have shorter telomeres, compared with unaffected family members, sporadic cases, and healthy controls [28]. He et al. [29] investigated the gene copy number and mRNA expression of different genes involved in telomere maintenance ( $P O T 1$ gene included) in 13 patients from six families with FNMTC observing no significant differences. Cantara et al. [15] studied POT1 and RAP1 genes by DHPLC in 66 patients from 38 families, implying that the majority of families had only two affected cases. Although they did not detect mutations in POT1, we consider it was not strong enough to rule out the implication of $P O T 1$ as a gene implied in hereditary TC because the analysis was done by DHPLC and the study was carried out mostly in families with only two cases. Given the high prevalence of TC in the general population, Charkes [30] estimated that about $62 \%$ of families with two cases affected by FNMTC can be phenocopies (two sporadic cases associated by chance); therefore, only $38 \%$ would be truly hereditary.
However, if there are three affected cases, the probability of being hereditary rises to $96 \%$. Consequently, we designed this multicentric study to analyze in selected FNMTC families (with 3 or more affected patients/family, and without any feature that made us suspect a well-known syndromic FNMTC) if the POT1 gene could be a putative susceptibility gene for non-syndromic FNMTC. Our enrolled families are representative of nonsyndromic FNMTC, with a very high probability of being hereditary, not only because of the important number of affected members, but also because they present similar clinical characteristics, as described before for other families with FNMTC, especially those with 3 or more affected members [31]: early age at diagnosis, lower female predominance, more tumor multifocality, more lymph node metastasis, and tumor aggressiveness and recurrence.

We performed WES analysis in four families and Sanger sequencing in the seven families. WES does not cover all the regions of POT1 gene (e.g., 5'UTR regions or large intronexon boundaries). On the other hand, WES analysis provided us complementary information, since it allowed us to rule out the presence of pathogenic mutations in other genes previously described as implicated in FNMTC. 
In our study, no germline potentially pathogenic mutations were detected in POT1 gene in seven families with FNMTC.

We did not find putative causative mutations in genes previously described as candidate genes for FNMTC in the four families studied by WES.

A limitation of the study is the relatively small number of families. However, it is difficult to recruit families with more than two members affected with NMTC, even with a multicentric collaboration. In fact, most scientific articles on this topic include a limited number of families analyzed, unless they include families with two affected members [15, 29].

In the multiple studies searching for causative genes of FNMTC in the literature, no reproducible results have been found. We hypothesize that FNMTC may be mainly a polygenic hereditary entity. This scenario would make it difficult to find a common link among the different affected families. Indeed, part of the families included in this study were included in a previous paper [32], where we suggest that non-syndromic FNMTC may be due to multiple mutations acting as risk alleles and modifier locus for FNMTC, in contrast with most hereditary cancers, in which only 2 or 3 high-penetrance causative genes have been described.

In conclusion, the absence of germline POT1 mutations in our set of TC families studied here does not completely exclude the possibility that $P O T 1$ mutations may be associated with an increased TC risk as part of the wide spectrum of POT1 cancers. However, our results minimize the chances of POT1 being a TC predisposition gene that would manifest itself in non-syndromic TC families.

Acknowledgments We are grateful to the patients and their families for their participation; and to their physicians for providing samples and clinical histories: F. Hanzu, J. Blanco, A. de Hollanda, and E. Ortega (H.Clínic); C. Villabona, J. Otero, and A. Simó (H. Bellvitge); M.C. Vilardell and A.M. Gutiérrrez (H.Althaia de Manresa); R. Casañ (H. de Xàtiva); B. León (H.de la Plana); R. Alfayate (H. General de Alicante); S. Martínez (H. de Elda); and C. Quirós (H.Mútua de Terrassa).

Funding Information This work was funded by the Fellowship 2015/ 2016 from Catalan Society of Endocrinology and Nutrition (Spain) to Josep Oriola.

\section{Compliance with Ethical Standards}

Conflict of Interest The authors declare that they have no conflict of interest.

Ethical Approval

All procedures performed in studies involving human participants were in accordance with the ethical standards of the institutional research committee (Ethics Committee of the Hospital Clínic of Barcelona, Spain; Reg. HCB/2016/0200) and with the 1964 Helsinki declaration and its later amendments or comparable ethical standards.

Informed Consent Informed consent was obtained from all individual participants included in the study.

\section{References}

1. Sahasrabudhe R, Stultz J, Williamson J et al (2016) The HABP2 G534E variant is an unlikely cause of familial non-medullary thyroid cancer. J Clin Endocrinol Metab 10:1098-1103. https://doi. org $/ 10.1210 /$ jc.2015-3928

2. Moses W, Weng J, Kebebew E (2011) Prevalence, clinicopathologic features, and somatic genetic mutation profile in familial versus sporadic non-medullary thyroid cancer. Thyroid 21:367-371. https://doi.org/10.1089/thy.2010.0256

3. Kebebew E (2008) Hereditary non-medullary thyroid cancer. World J Surg 32:678-682. https://doi.org/10.1007/s00268-007-9312-z

4. Rowland KJ, Moley JF (2015) Hereditary thyroid cancer syndromes and genetic testing. J Surg Oncol 111:51-60. https://doi. org/10.1002/jso.23769

5. Vriens MR, Suh I, Moses W, Kebebew E (2009) Clinical features and genetic predisposition to hereditary non-medullary thyroid cancer. Thyroid 19:1343-1349. https://doi.org/10.1089/thy.2009.1607

6. Malchoff CD, Sarfarazi M, Tendler B et al (2000) Papillary thyroid carcinoma associated with papillary renal neoplasia: genetic linkage analysis of a distinct heritable tumor syndrome. J Clin Endocrinol Metab 85:1758-1764. https://doi.org/10.1210/jcem.85.5.6557

7. Suh I, Filetti S, Vriens MR et al (2009) Distinct loci on chromosome 1q21 and 6q22 predispose to familial non-medullary thyroid cancer: a SNP array-based linkage analysis of 38 families. Surgery 146 : 1073-1080. https://doi.org/10.1016/j.surg.2009.09.012

8. Gudmundsson J, Sulem P, Gudbjartsson DF et al (2009) Common variants on $9 \mathrm{q} 22.33$ and $14 \mathrm{q} 13.3$ predispose to thyroid cancer in European populations. Nat Genet 41:460-464. https://doi.org/10. 1038/ng.339

9. Gara SK, Jia L, Merino MJ et al (2015) Germline HABP2 mutation causing familial nonmedullary thyroid cancer. N Engl J Med 373: 448-455. https://doi.org/10.1056/NEJMoa1502449

10. Ye F, Gao H, Xiao L et al (2019) Whole exome and target sequencing identifies MAP2K5 as novel susceptibility gene for familial non-medullary thyroid carcinoma. Int J Cancer 144:1321-1330. https://doi.org/10.1002/ijc.31825

11. Pereira JS, da Silva JG, Tomaz RA et al (2015) Identification of a novel germline FOXE1 variant in patients with familial nonmedullary thyroid carcinoma (FNMTC). Endocrine 49:204-214. https://doi.org/10.1007/s12020-014-0470-0

12. Weeks AL, Wilson SG, Ward L, Goldblatt J, Hui J, Walsh JP (2016) HABP2 germline variants are uncommon in familial nonmedullary thyroid cancer. BMC Med Genet 17:60. https://doi.org/10.1186/ s12881-016-0323-1

13. Colombo C, Fugazzola L, Muzza M et al (2018) Letter regarding the article: "Multiple HABP2 variants in familial papillary thyroid carcinoma: contribution of a group of 'thyroid-checked' controls" by Kern et al. Eur J Med Genet 61:104-105. https://doi.org/10. 1016/j.ejmg.2017.07.012

14. Liu R, Xing M (2016) TERT promoter mutations in thyroid cancer. Endocr Relat Cancer 23(3):143-155. https://doi.org/10.1530/ERC15-0533

15. Cantara S, Capuano S, Capezzone M et al (2012) Lack of mutations of the telomerase RNA component in familial papillary thyroid cancer with short telomeres. Thyroid 22(4):363-368. https://doi. org/10.1089/thy.2011.0109

16. Wong K, Robles-Espinoza CD, Rodriguez D et al (2019) Association of the POT1 germline missense variant p.I78T with familial melanoma. JAMA Dermatol 155(5):604-609. https://doi. org/10.1001/jamadermatol.2018.3662

17. Bagcchi S (2015) POT1: a genetic link for familial glioma. Lancet Oncol 16(1):2. https://doi.org/10.1016/S1470-2045(14)71178-7

18. Calvete O, Martinez P, Garcia-Pavia P et al (2015) A mutation in the POT1 gene is responsible for cardiac angiosarcoma in TP53- 
negative Li-Fraumeni-like families. Nat Commun 6:8383. https:// doi.org/10.1038/ncomms9383

19. Wilson TL, Hattangady N, Lerario AM et al (2017) A new POT1 germline mutation-expanding the spectrum of POT1-associated cancers. Familial Cancer 16(4):561-566. https://doi.org/10.1007/ s10689-017-9984-y

20. Potrony M, Puig-Butille JA, Ribera-Sola M et al (2019) POT1 germline mutations but not TERT promoter mutations are implicated in melanoma susceptibility in a large cohort of Spanish melanoma families. Br J Dermatol 181(1):105-113. https://doi.org/10. 1111/bjd.17443

21. Calvete O, Garcia-Pavia P, Domínguez F et al (2017) The wide spectrum of POT1 gene variants correlates with multiple cancer types. Eur J Hum Genet 25(11):1278-1281. https://doi.org/10. 1038/ejhg.2017.134

22. Saporito D, Brock P, Hampel H et al (2018) Penetrance of a rare familial mutation predisposing to papillary thyroid cancer. Familial Cancer 17(3):431-434. https://doi.org/10.1007/s10689-017-0048-0

23. Hińcza K, Kowalik A, Kowalska A (2019) Current knowledge of germline genetic risk factors for the development of non-medullary thyroid cancer. Genes (Basel) 10(7):E482. https://doi.org/10.3390/ genes 10070482

24. Santos LS, Costa-Gomes B, Bastos HN et al (2019) Thyroid cancer: the quest for genetic susceptibility involving DNA repair genes. Genes (Basel) 10(8):E586. https://doi.org/10.3390/genes 10080586

25. Goggins W, Daniels GH, Tsao H (2006) Elevation of thyroid cancer risk among cutaneous melanoma survivors. Int J Cancer 118(1): 185-188. https://doi.org/10.1002/ijc.21300
26. Liu X, Bishop J, Shan Y et al (2013) Highly prevalent TERT promoter mutations in aggressive thyroid cancers. Endocr Relat Cancer 20(4):603-610. https://doi.org/10.1530/ERC-13-0210

27. Marques IJ, Moura MM, Cabrera R et al (2017) Identification of somatic TERT promoter mutations in familial nonmedullary thyroid carcinomas. Clin Endocrinol 87(4):394-399. https://doi.org/ 10.1111/cen.13375

28. Capezzone M, Cantara S, Marchisotta S et al (2008) Short telomeres, telomerase reverse transcriptase gene amplification, and increased telomerase activity in the blood of familial papillary thyroid cancer patients. J Clin Endocrinol Metab 93:3950-3957. https://doi. org $/ 10.1210 /$ jc.2008-0372

29. He M, Bian B, Gesuwan K et al (2013) Telomere length is shorter in affected members of families with familial non-medullary thyroid cancer. Thyroid 23(3):301-307. https://doi.org/10.1089/thy.2012. 0270

30. Charkes ND (2006) On the prevalence of familial nonmedullary thyroid cancer in multiply affected kindreds. Thyroid 16:181-186. https://doi.org/10.1089/thy.2006.16.181

31. Wang X, Cheng W, Li J et al (2015) Familial non-medullary thyroid carcinoma is a more aggressive disease: a systematic review and meta-analysis. Eur J Endocrinol 172:253-262. https://doi.org/10. 1530/EJE-14-0960

32. Orois A, Gara SK, Mora M et al (2019) NOP53 as a candidate modifier locus for familial non-medullary thyroid cancer. Genes (Basel) 10(11):E899. https://doi.org/10.3390/genes10110899

Publisher's Note Springer Nature remains neutral with regard to jurisdictional claims in published maps and institutional affiliations. 